\title{
SUMMANDS OF PERMUTATION LATTICES FOR FINITE GROUPS
}

\author{
GERALD CLIFF AND ALFRED WEISS
}

(Communicated by Maurice Auslander)

\begin{abstract}
Let $G$ be a finite group. An effective criterion is given for a $\mathbf{Z} G$ lattice to be a direct summand of a permutation lattice.
\end{abstract}

Let $G$ be a finite group. A $\mathbf{Z} G$-lattice is by definition a $\mathbf{Z} G$-module which is free and finitely generated as a $\mathbf{Z}$-module. Such a module is called a permutation lattice if it has a $\mathbf{Z}$-basis which is permuted by $G$, and it is called invertible if it is a direct summand, as a $\mathrm{Z} G$-module, of a permutation lattice. These modules have been studied in recent years in connection with questions of rationality of field extensions and function fields of algebraic tori. (An excellent survey is Swan [4]; invertible modules are also called permutation projective, for instance in [2].) Our aim is to give the following criterion for a $\mathbf{Z G}$-lattice to be invertible.

Theorem. $A \mathrm{ZG}$-lattice $M$ is invertible if and only if it satisfies $\mathrm{I}_{p}$ for all primes $p$ and also II, where

$I_{p}$ For a Sylow p-subgroup $P$ of $G$ the restriction $(M / p M)_{P}$ of $M / p M$ to $P$ is a permutation module for $\mathbf{F}_{p} P$.

II For a Sylow 2-subgroup $P$ of $G$ the dimensions $\operatorname{dim} F_{2}(M / 2 M)^{P}$ and $\operatorname{dim}_{\mathbf{Q}}(\mathbf{Q} \otimes M)^{P}$ of $P$-fixed points are equal.

We will prove this by first reducing to the case of a $p$-group, then replacing $\mathbf{Z}$ by the $p$-adic integers $\mathbf{Z}_{p}$; for odd primes, the result will follow by using Lemma 3 below. For $p=2$, Lemma 3 does not hold, and to complete the proof of the theorem we use a result of Weiss [5, Theorem 3]. In an earlier version of this work, we had used Weiss' theorem for all primes; we thank the referee who indicated to us that it could be avoided for odd primes. For results related to Lemmas 1 and 2 see Dress [2].

Lemma 1. Let $M$ be a $\mathrm{ZG}$-lattice. Then $M$ is invertible if and only if $M_{P}$ is an invertible $\mathrm{ZP}$-lattice for each Sylow p-subgroup $P$ of $G$ for all primes $p$.

Received by the editors March 31, 1989 and, in revised form, November 30, 1989.

1980 Mathematics Subject Classification (1985 Revision). Primary 20C10, 20C11; Secondary 11 R32.

This research was supported in part by a grant from NSERC of Canada. 
Proof. Let $\mathscr{P}$ be a set containing one Sylow subgroup for each prime dividing the order of $G$. For each $P \in \mathscr{P}$ there is a $\mathbf{Z} G$-epimorphism $\phi_{P}: \operatorname{ind}_{P}^{G}\left(M_{P}\right) \rightarrow$ $M$ such that $\phi_{P}(g \otimes m)=g m$, for $g \in G$ and $m \in M$. Let

$$
\phi=\bigoplus_{P \in \mathscr{P}} \phi_{P}: \bigoplus_{P \in \mathscr{P}} \operatorname{ind}_{P}^{G}\left(M_{P}\right) \rightarrow M
$$

be the sum of the maps $\phi_{P}$. Since the indices $|G: P|, P \in \mathscr{P}$, have greatest common divisor 1 , we can find integers $a_{P}, P \in \mathscr{P}$, with $\sum_{P \in \mathscr{P}} a_{P}|G: P|=1$. Then the epimorphism $\phi$ is split by the $\mathbf{Z} G$-homomorphism $\psi$ defined by

$$
\psi(m)=\sum_{P \in \mathscr{P}} a_{P} \sum_{g \in G / P} g \otimes g^{-1} m, \quad m \in M .
$$

Therefore, $M$ is isomorphic to a direct summand of $\bigoplus_{P \in \mathscr{P}} \operatorname{ind}_{P}^{G}\left(M_{P}\right)$. The lemma now follows easily.

Lemma 2. Let $P$ be a p-group for a prime $p$, and let $M$ be a $\mathrm{ZP}$-lattice. Then $M$ is invertible if and only if $\mathbf{Z}_{p} \otimes M$ is a permutation lattice for $\mathbf{Z}_{p} P$.

Proof. Suppose that $M$ is invertible; then $\mathbf{Z}_{p} \otimes M$ is isomorphic to a direct summand of a permutation $\mathrm{Z}_{p} P$-lattice. A transitive permutation $\mathrm{Z}_{p} P$-lattice is indecomposable (which follows, for instance, from Green's indecomposability theorem $[1,19.22])$, so $\mathbf{Z}_{p} \otimes M$ is a permutation lattice, by Krull-Schmidt.

Conversely, suppose that $\mathbf{Z}_{p} \otimes M$ is a permutation module. We may find a permutation $\mathrm{Z} P$-lattice $L$ with $\mathrm{Z}_{p} \otimes L \cong \mathrm{Z}_{p} \otimes M$. Since $P$ is $p$-group, then $L$ and $M$ are in the same genus, in the sense that their completions over all primes are isomorphic, by [1, 31.2(ii) and 27.1]. By Roiter's Lemma [1, 31.6] there is an exact $\mathrm{ZP}$-sequence

$$
0 \rightarrow L \rightarrow M \rightarrow T \rightarrow 0,
$$

where $T$ is finite of order prime to $p$. Let $F$ be a free $\mathrm{Z} P$-module mapping onto $T$, and consider the exact sequence

$$
0 \rightarrow K \rightarrow F \rightarrow T \rightarrow 0,
$$

where $K$ is the kernel of the map from $F$ to $T$. Then by Roiter's version of Schanuel's Lemma $[1,31.8]$ we have $M \oplus K \cong L \oplus F$ so $M$ is isomorphic to a direct summand of the permutation lattice $L \oplus F$. This completes the proof.

Lemma 3. Let $P$ be a p-group, for a prime $p>2$, and let $M$ be a $\mathrm{Z}_{p} P$-lattice. Then $M$ is a permutation lattice if and only if $M / p M$ is a permutation module for $\mathbf{F}_{p} P$.

Proof. Suppose that $M / p M$ is a permutation module. We claim:

$$
H^{1}(Q, M)=0 \text { for all subgroups } Q \text { of } P \text {. }
$$

We prove this by induction on $|P|$. If $P$ has order $p$, then there are only three isomorphism types of indecomposable $\mathrm{Z}_{p} P$-lattices $M$ (see [1, p. 729]): the trivial module, the free module $\mathrm{Z}_{p} P$, and the augmentation ideal of $\mathrm{Z}_{p} P$. 
For the first two types $H^{1}(P, M)=0$, but since the third is not a permutation module $\bmod p$ because $p>2$, it is not considered. Thus the claim holds in this case. For $|P|>p$, only $H^{1}(P, M)=0$ must be shown, because the induction hypothesis handles proper subgroups of $P$. Let $Q$ be a central subgroup of $P$ of order $p$. The exact sequence $0 \rightarrow M \stackrel{p}{\rightarrow} M \rightarrow M / p M \rightarrow 0$ gives rise to

$$
0 \rightarrow M^{Q} \stackrel{p}{\rightarrow} M^{Q} \rightarrow(M / p M)^{Q} \rightarrow H^{1}(Q, M) .
$$

Since $H^{1}(Q, M)=0$ by induction, then $(M / p M)^{Q} \cong M^{Q} / p\left(M^{Q}\right)$. Since $M / p M$ is a permutation module for $P$, then $(M / p M)^{Q}$ is a permutation module for $P / Q$. (It has as $\mathbf{F}_{p}$-basis the $Q$-orbit sums of a $P$-permutation $\mathbf{F}_{p}$-basis of $M / p M$.) By induction the claim applied to the $\mathbf{Z}_{p}(P / Q)$-lattice $M^{Q}$ yields $H^{1}\left(P / Q, M^{Q}\right)=0$. Then the inflation-restriction sequence [3, p. 125]

$$
0 \rightarrow H^{1}\left(P / Q, M^{Q}\right) \rightarrow H^{1}(P, M) \rightarrow H^{1}(Q, M)^{P / Q}
$$

implies that $H^{1}(P, M)=0$, and the claim is proved.

Now let $\mathscr{B}$ be a basis of $M / p M$ which is permuted by $P$. Write $\mathscr{B}=\cup \mathscr{C}$ as a disjoint union of orbits, and take an element $y$ in some orbit $\mathscr{C}$. Let $Q$ be the stabilizer in $P$ of $y$, and write $P=\cup_{t \in \mathcal{T}} t Q$ as a union of cosets. By the claim and $(*)$, there exists $x \in M^{Q}$ such that $y=x+p M$. Define $\mathscr{C}^{\prime}=\{t x: t \in \mathscr{T}\}$, and let $\mathscr{B}^{\prime}=\cup \mathscr{C}^{\prime}$. Then $\mathscr{B}^{\prime}$ is permuted by $P$, and $\mathscr{B}^{\prime}$ is a $\mathbf{Z}_{p}$-basis of $M$ by Nakayama's Lemma, since $\mathscr{B}^{\prime}$ reduced modulo $p M$ is $\mathscr{B}$. Therefore, $M$ is indeed a permutation $\mathrm{Z}_{p} P$-lattice. This proves the lemma, since the converse is obvious.

Proof of theorem. By Lemma 1 we may assume that $G$ is a $p$-group $P$ for some prime $p$. Then Lemma 2 transfers the problem of deciding if a $\mathbf{Z} P$-lattice $M$ is invertible to deciding if the $\mathrm{Z}_{p} P$-lattice $L=\mathrm{Z}_{p} \otimes M$ is a permutation lattice, because $M / p M \cong L / p L$. In case $p=2$ the condition II can be translated to $L$ because $\mathbf{Q}_{2} \otimes L \cong \mathbf{Q}_{2} \otimes_{\mathbf{Q}}(\mathbf{Q} \otimes M)$ and $\mathbf{Q}_{2} \otimes_{\mathbf{Q}} *$ does not change fixed point dimensions. If $p>2$ the theorem follows immediately from Lemma 3.

Finally if $p=2$ and $L$ satisfies $I_{2}$ and II then [5, Theorem 3] tells us that $L \cong \bigoplus_{i=1}^{n} \operatorname{ind}_{P_{i}}^{P}\left(\left(\mathbf{Z}_{2}\right)_{\chi_{i}}\right)$ for certain homomorphisms $\chi_{i}: P_{i} \rightarrow\{ \pm 1\}$, where each $P_{i}$ is a subgroup of $P$ and $\left(\mathbf{Z}_{2}\right)_{\chi_{i}}$ denotes the $\mathbf{Z}_{2} P_{i}$-lattice with underlying $\mathbf{Z}_{2}$-module $\mathbf{Z}_{2}$ and $P_{i}$ acting via $\chi_{i}$. Then $L / 2 L \cong \bigoplus_{i=1}^{n} \operatorname{ind}_{P_{i}}^{P}\left(\mathbf{F}_{2}\right)$, and so $\operatorname{dim}_{F_{2}}(L / 2 L)^{P}=n$, the number of homomorphisms $\chi_{i}$. On the other hand, $\operatorname{dim}_{\mathbf{Q}_{2}}\left(\mathbf{Q}_{2} \otimes_{\mathbf{Z}_{2}} L\right)^{P}$ can be computed from the character $\ddot{\zeta}$ of $L:$ it is the number of times the trivial character occurs in $\xi$, which is by Frobenius reciprocity the number of homomorphisms $\chi_{i}$ which are trivial. By hypothesis II, all the $\chi_{i}$ must be trivial, so $L$ is a permutation lattice in this case too. Since the converse is obvious, the proof of the theorem is complete.

Note that the theorem does not hold without hypothesis II: $M$ could be the rank $1 \mathbf{Z} G$-lattice $\mathbf{Z}$ on which some element of $G$ of even order acts by 
multiplication by -1 . We may define a signed permutation lattice for $\mathrm{Z} G$ to be a lattice which has a Z-basis $\left\{m_{i}\right\}$ such that for each $g$ in $G, g m_{i}= \pm m_{j}$ for some $j$. It is not difficult to see that our proof of the theorem can be modified (indeed simplified) to prove the following result.

Theorem. $A$ ZG-lattice $M$ is a direct summand of a signed permutation lattice if and only if it satisfies $\mathrm{I}_{p}$ for all primes $p$.

\section{REFERENCES}

1. C. W. Curtis and I. Reiner, Methods of representation theory. I, Wiley-Interscience, New York, 1981.

2. A. Dress, The permutation class group of a finite group, J. Pure Appl. Algebra 6 (1975), $1-12$.

3. J.-P. Serre, Corps locaux, Hermann, Paris, 1968.

4. R. G. Swan, Noether's problem in Galois theory, Emmy Noether in Bryn Mawr, SpringerVerlag, New York and Berlin, 1983, pp. 21-40.

5. A. Weiss, Rigidity of p-adic p-torsion, Ann. of Math. 127 (1988), 317-332.

Department of Mathematics, University of Alberta, Edmonton, Alberta t6G 2G1 CANADA 